\title{
Controlled release profiles of dipyridamole from biodegradable microspheres on the base of poly(3-hydroxybutyrate)
}

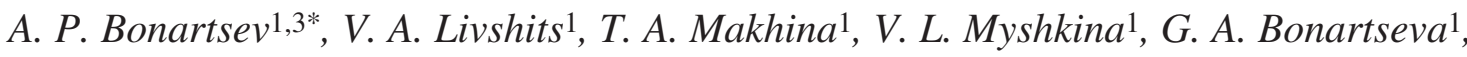 \\ A. L. Iordanskii ${ }^{1,2}$ \\ ${ }^{1}$ Laboratory of biochemistry of nitrogen fixation, A. N. Bach's Institute of Biochemistry, Russian Academy of Sciences, \\ Leninskii prosp. 33, 119071 Moscow, Russia \\ 2Laboratory of transport phenomena in polymers, N. N. Semenov's Institute of Chemical Physics, Russian Academy of \\ Sciences, Kosygin str. 4, 119991 Moscow, Russia \\ ${ }^{3}$ Laboratory of enzyme chemistry, Faculty of Biology, Moscow State University, Leninskie gory 1-12, 119992 Moscow, \\ Russia
}

Received 19 July 2007; accepted in revised form 15 October 2007

\begin{abstract}
Novel biodegradable microspheres on the base of poly(3-hydroxybutyrate) (PHB) designed for controlled release of antithrombotic drug, namely dipyridamole (DPD), have been kinetically studied. The profiles of release from the microspheres with different diameters $4,9,63$, and $92 \mu \mathrm{m}$ present the progression of nonlinear and linear stages. Diffusionkinetic equation describing both linear (PHB hydrolysis) and nonlinear (diffusion) stages of the DPD release profiles from the spherical subjects has been written down as the sum of two terms: desorption from the homogeneous sphere in accordance with diffusion mechanism and the zero-order release. In contrast to the diffusivity dependence on microsphere size, the constant characteristics $(k)$ of linearity are scarcely affected by the diameter of PHB microparticles. The view of the kinetic profiles as well as the low rate of DPD release are in satisfactory agreement with kinetics of weight loss measured in vitro for the PHB films. Taking into account kinetic results, we suppose that the degradation of both films and PHB microspheres is responsible for the linear stage of DPD release profiles. In the nearest future, combination of biodegradable PHB and DPD as a representative of proliferation cell inhibitors will give possibility to elaborate the novel injectable therapeutic system for a local, long-term, antiproliferative action.
\end{abstract}

Keywords: biodegradable polymers, poly(3-hydroxybutyrate), microspheres, controlled drug release, diffusion

\section{Introduction}

For the last three decades the intensive research studies and developments of polymer systems for controlled release of pharmaceuticals have been carried out [1-3]. At curative dose the prolonged delivery of drugs from the systems into organism permits to eliminate the shortcomings in peroral, injectable, aerosol, and the other traditional methods of drug administration. Among those shortcomings hypertoxicity, instability, pulsative character of rate delivery, ineffective expenditure of drugs should be pointed out. Alternatively, applications of therapeutical polymer systems provide orderly and purposefully the deliverance for an optimal dose of agent that is very important at therapy of acute or chronic diseases [4]. At treatment of cardiovascular and other diseases one of the perspective classes of drugs is the class of inhibitors of cell proliferation (ICP). In particular, pharmaceuticals on the base of ICP are widely used for preventing

*Corresponding author, e-mail: bonar@inbi.ras.ru

(C) BME-PT and GTE 
the spread of restenosis after operations in cardiovascular surgery $[5,6]$. In this relation, it has been recently shown that a wide-spread antithrombotic drug dipyridamole (DPD) inhibits effectively the cell proliferation, but the DPD advantages the minority of negative by-effects $[7,8]$ in contradistinction to most of ICP. In accordance with this argument DPD has been picked over as the effector for designing polymer therapeutic system for controlled drug delivery. Quit recently Zhu and coworkers [9] have designed the DPD controlled release polymer system on the base of microspheres composed of polylactide - polyglicolide copolymers (PLGA). They have clearly shown that DPD release includes two stages: the diffusion stage and the subsequent stage of hydrolytic degradation of PLGA. It is worth to note that the use of implants consisting of polylactides, polyglicolides and their copolymers is not without a number of sequelae related with the chronic inflammatory reactions in tissue [10-14].

Instead of PLGA applications, we propose alternatively to use bacterial poly(3-hydroxybutyrate) (PHB) [15]. Recently PHB and its copolymers are a focus of attention as biodegradable and biocompatible materials for biomedicine. The combination of physical and biological properties promote the use of this polymer for design and elaboration of different medical devices such as surgical sutures, repair patches, vascular prostheses, stents, periodontal membranes, orthopaedic implants, wound dressings etc. [16]. Besides, the ability of PHB to encapsulation and further release of drugs permits the use of PHB to design novel therapeutic systems [15]. Taking into account the above arguments, the objects of this paper are the production of PHB microspheres loaded with the DPD as well as the study of kinetic profile of the drug release in cardiovascular therapy. In the nearest future, combination of biodegradable PHB and DPD as a representative of proliferation cell inhibitors will give possibility to design an injectable therapeutic system for a local, long-term, antiproliferative action.

\section{Materials and methods}

\subsection{PHB production}

The PHB strain used in this work (Azotobacter chroococcum $7 B$ ) was capable of synthesizing PHB in an amount of up to $80 \%$ on the dry weight basis of the cells. A collection of strains of the genus Azotobacter were maintained on Ashbey's medium. To achieve cellular PHB hyperproduction, the culture of the Azotobacter strain was grown on Burke's medium in excess of content of the carbon source [17]. The process of isolation and purification from the biomass of $A$. chroococcum $7 B$ included the following stages: dissolution of PHB in chloroform by shaking at $37^{\circ} \mathrm{C}$ for $12 \mathrm{~h}$, separation of the PHB solution from the cell residue by filtration, isolation of the PHB by isopropanol precipitation, and repeated its dissolution in chloroform followed by isopropanol precipitation and drying at $60^{\circ} \mathrm{C}$. The MW of the polymer was determined by viscosimetry. Measurements of viscosity in the PHB - chloroform solutions were performed at $30^{\circ} \mathrm{C}$. The MW was calculated using the MarkHouwink-Kuhn equation $[\eta]=7.7 \cdot 10^{-5} \cdot \mathrm{M}^{0.82}[18]$. The chemical structure of PHB, the type of its crystal lattice, and its crystallinity (0.74) were previously determined using the methods of differential scanning calorimetry, IR Fourier spectroscopy, and crystal X-ray structure analysis [19].

\subsection{Microsphere preparation and characterization}

Dipyridamole-incorporated PHB microspheres were prepared using an oil-in-water emulsion method [9]. PHB polymer with molecular weight (MW) of $485 \mathrm{kDa}$ was used for microspheres preparation. DPD and PHB in proportions of 1:4 were dissolved in $8 \mathrm{ml}$ of chloroform. The solution was added drop-wise to $300 \mathrm{ml}$ of PVA solution $(0.4-1.2 \% \mathrm{w} / \mathrm{v})$ while stirred. The mixture was homogenized for $2 \mathrm{~h}$ at 600-2000 rpm using an overhead stirrer RZR 2021 (Heidolph, Germany) or at 20000 using a homogenizer SilentCruisher M (Heidolph, Germany). After complete chloroform evaporation the produced solid microspheres were divided by glass filter with various pore sizes. The final product was obtained by centrifugation (5702 R centrifuge, Eppendorf, Germany), washing with distilled water. The centrifugation and washing processes were repeated at least five times to completely remove PVA and DPD adsorbed onto the microspheres surface. Then the microspheres were dried in thermostat at $60^{\circ} \mathrm{C}$ and gently powdered. Encapsulation efficiency of microspheres was calculated from the ratio of the encapsulated 
drug amount in microspheres to the total drug amount used for preparation.

For the microspheres with the highest diameter (group 4) we duplicated the concentration of DPD in order to enhance the rate of release. (The rate of release is decreased with the increase of sphere diameter). The concentration increase was required for the sake of adjustment of all kinetic profiles including the largest microspheres in the same time scale ( $x$-axis). The amount of loaded DPD was determined by dissolving the microspheres in chloroform followed by spectrophotometric analysis with UV-spectrophotometer DU-650 (Beckman Coulter, USA) at maximums of DPD absorption (293 and $415 \mathrm{~nm}$ ). The preparation of PHB films for loss of polymer mass presented in Figure 4 has been described in our preliminary paper [15].

\subsection{In vitro release}

Controlled release of DPD from PHB microspheres was carried out at conditions in vitro: in phosphatebuffered saline ( $\mathrm{pH} \mathrm{7.4)}$ at $37^{\circ} \mathrm{C}$. Eight lots of each group of about $5 \mathrm{mg}$ of DPD-loaded PHB microspheres were used as drug depot. $4 \mathrm{ml}$ of release medium (phosphate-buffered saline, $\mathrm{pH}$ 7.4) was added to each drug depot in the vial to maintain sink conditions. The mixture was agitated at $50 \mathrm{rpm}$ at magnetic stirrer MS-01 (Elmi, Latvia) in a thermostat TC-1/20 (Russia) at $37^{\circ} \mathrm{C}$. At specific time points, release medium was sampled completely by centrifugation of mixture at $14000 \mathrm{rpm}(5702 \mathrm{R}$ centrifuge, Eppendorf, Germany) and assayed for DPD using the spectroscopic method. The release kinetics was then determined. The removed release medium was replaced with an equal volume of fresh medium after each sampling and was added to deposited microspheres. Residual content of DPD in microspheres was also measured by spectrophotometric technique. The conditions of experiment (stirring rate, volume and concentration of working solutions) for obtaining of DPD-loaded PHB microspheres with different diameter are collected in Table 1. From this table we can see that the stirring rate is a dominating factor affecting the sphere diameter. The weight loss of polymer films was determined by drying and weighting of the samples after their exposition in the buffer as it was described in [15].

\section{Results and discussion}

As a drug vehicle of DPD, we have used the microspheres produced from PHB solution by the oil-in-water emulsion method described in Experimental part. The pioneer work devoted to PHB microsphere preparation have been presented in [18]. Figure 1 presents a typical microphotograph of spherical samples with diameter $63 \mu \mathrm{m}$ loaded by DPD. As can be seen in this figure, the PHB subjects have the spherical form without visible defects. They are adequately homogeneous and do not have visible inclusions or voids. Besides, the yellow DPD has slightly colored the interior of the microspheres; therefore, as well as Kassab et al. [20], we assume a uniform distribution of the drug throughout the volume of a microsphere.

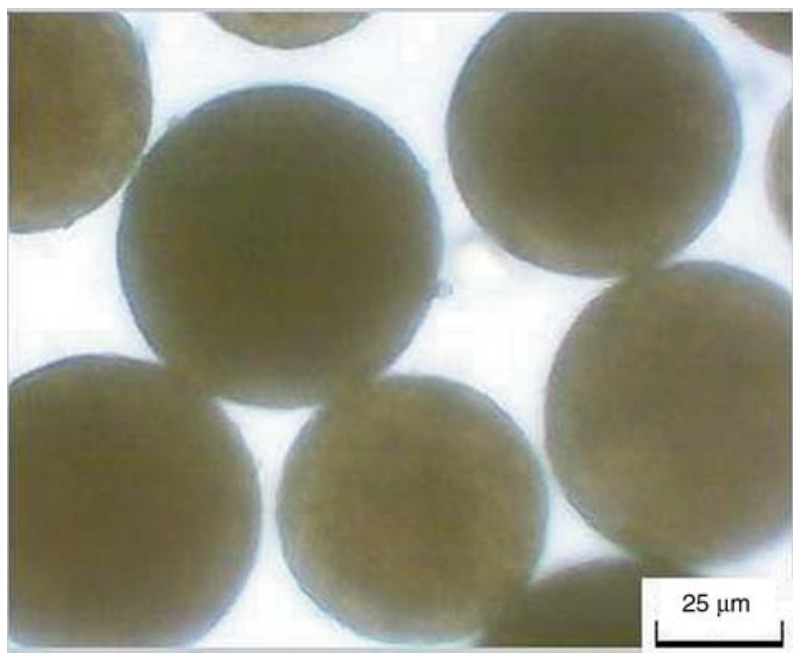

Figure 1. Microphotographs of PHB microspheres loaded by DPD with diameters $63 \mu \mathrm{m}$

Table 1. The conditions for producing PHB microspheres of different diameter loaded with definite content of DPD

\begin{tabular}{|c|c|c|c|c|c|c|}
\hline $\begin{array}{c}\text { Group } \\
\#\end{array}$ & $\begin{array}{c}\text { Mass of DPD in } \\
\text { working solution } \\
{[\mathbf{m g}]}\end{array}$ & $\begin{array}{c}\text { Mass of PHB in } \\
\text { working solution } \\
{[\mathbf{m g}]}\end{array}$ & $\begin{array}{c}\text { PVA } \\
\text { concentration } \\
{[\%(\mathbf{w} / \mathbf{w})]}\end{array}$ & $\begin{array}{c}\text { Stirring } \\
\text { rate } \\
{[\mathbf{r p m}]}\end{array}$ & $\begin{array}{c}\text { Diameter of } \\
\text { microspheres } \\
\mathbf{m k m} \pm \mathbf{S D}\end{array}$ & $\begin{array}{c}\text { DPD content in } \\
\text { PHB microspheres } \\
{[\mathbf{\%}(\mathbf{w} / \mathbf{w})]^{*}}\end{array}$ \\
\hline 1 & 24 & 96 & 0.8 & 2000 & $3.6 \pm 2.4$ & $4.8 \pm 0.4$ \\
\hline 2 & 24 & 96 & 0.6 & 1000 & $18.7 \pm 2.9$ & $5.2 \pm 0.4$ \\
\hline 3 & 24 & 96 & 0.4 & 600 & $62.7 \pm 6.6$ & $4.9 \pm 0.4$ \\
\hline 4 & 47 & 100 & 0.6 & 500 & $91.7 \pm 15.4$ & $11.0 \pm 0.5$ \\
\hline
\end{tabular}

*Averaged values for ten samples with standard deviation 


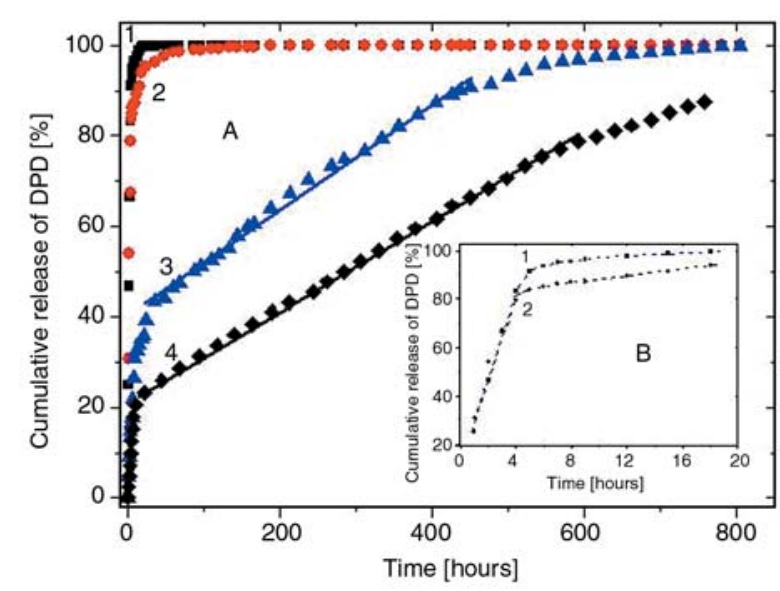

Figure 2. Kinetics profiles of DPD release from PHB microspheres in vitro (phosphate buffer, $37^{\circ} \mathrm{C}$ ). A: General view of kinetic curves for the microspheres with different diameters: 4(1), 19(2), 63(3), and 92(4) $\mu \mathrm{m}$. The lines show the second stage of release following the zero-order equation. B: Details of the curves for the microspheres with the smaller diameters: 4(1), 19(2)

The kinetic profiles of DPD release into phosphate buffer for the microspheres with different diameters are presented in Figure 2. The curves 3 and 4 referring to the series with the bigger diameters 63 and $92 \mu \mathrm{m}$, respectively, have two distinctive ranges: the initial range where fast release is observed and the following one where the constant rate is exhibited for a sufficiently long time of release (several hundreds of hours). For the microspheres with a relatively small diameter $(19 \mu \mathrm{m}$, curve 2), we may also point out a small linear part of the drug release curve for 6-21 h interval, but for the smallest samples (diameter is as small as $4 \mu \mathrm{m}$, curve 1) the linear range is practically missing.

The similar profiles of drug release had been observed and have been well described for the microspheres on the base of PLGA and loaded by 5-fluorouracil [21]. The difference between the kinetic profiles between PHB and PLGA microspheres is that the copolymer microspheres have the third kinetic range when at the final stage the abrupt emission of 5-fluorouracil comes about. Most recently, in our works describing release profiles of DPD [15], indomethacin [15], and 5-nitrofurfuriliden semicarbozone [22] from the films of PHB, we have shown that for all three drugs the kinetic curves of release have the initial diffusion stage which smoothly transforms to the linear stage. However, the third abrupt stage was absent for all systems for several weeks. Taking into account generally the Sipman's three-stage model of release [21, 23, 24], we note that for the PHB microspheres having a higher hydrolysis resistance as compared with PLGA, final stage of PHB network degradation and, hence, at the given conditions in vitro a drastic DPD discharge has not attained (see Figure 2). At the same time, reposing on the literature data and our experiments obtained for the 'PHB films - drug' systems [15, 22] we assume that the linear part of the kinetic curves for both the films and the microspheres could be interpreted rather as the start of zero-order hydrolytic reaction for PHB macromolecules.

Diffusion-kinetic equation describing both linear (hydrolysis) and nonlinear (diffusion) stages of the DPD release profiles from the spherical subjects could be written down as the sum of two terms : desorption from the homogeneous sphere in accordance with diffusion mechanism (the first summand involving square brackets in Equation (1a)) and the zero-power release (the addend in this equation):

$$
\frac{\partial G_{t}}{\partial t}=D\left[\frac{\partial^{2} G_{t}}{\partial z^{2}}+\left(\frac{2}{z}\right)\left(\frac{\partial G_{t}}{\partial z}\right)\right]+k
$$

Here $D$ is the constant (or averaged) diffusion coefficient of the drug in the polymer microspheres, $\left[\mathrm{cm}^{2} / \mathrm{sec}\right] ; G_{t}(z, t), \partial G_{t} / \partial z, \partial \mathrm{Gt} / \partial \mathrm{t}$ and $\partial^{2} G_{t} / \partial \mathrm{z}^{2}$ are the current drug concentration [\%], the first and second derivatives of $G_{t}$ with respect to $t$ (time) or $z$ (spherical coordinate of diffusion) respectively; and $k$ is the zero-order constant. Following Crank [25], transformation of Equation (1a) into the conventional diffusion equation (1b) for spheres has been performed by introduction of new variables $C_{t} \equiv G_{t}-k t$ and $u=C_{t} \cdot z$ :

$$
\frac{\partial u}{\partial t}=D\left[\frac{\partial^{2} u}{\partial z^{2}}\right]
$$

The boundary and initial conditions for solution of differential Equation (1a) have been written by standard way. At $t=0, C_{0}=$ const in the interval $0<z<R$, and at $t>0$ and $z=R$ we have $C_{0}=0$, at $t>0$ and $z=0, u=C_{t} \cdot z=0$.

In accordance with our approach, at any time of release the balance of the drug masses in the surrounding medium is fulfilled as Equation (2):

$G_{m t}=M_{t}+M_{H t}$ 
here $G_{m t}$ is the total amount of DPD [mg] released from the microspheres by the time $t ; M_{t}$ is the portion of DPD mass released via the diffusion process; and $M_{H t}$ is the other portion of DPD mass released via degradation mechanism.

It is common knowledge that solution of Equation (1b) has two variations, namely for relatively small time of diffusion when $M_{t} / M_{\infty} \leq 0.5$ approximately and for relatively long time when the condition $M_{t} / M_{\infty}>0.5$ is fulfilled. In the first instance the approximation (3) is correct [25]:

$\frac{M_{t}}{M_{\infty}}=6\left[\left(\frac{D_{\alpha} t}{\pi^{2} R^{2}}\right)^{0.5}\right]$

where $M_{t}, M_{\infty}$ are cumulative weights of the drug desorbed via diffusion by the time $t$ and infinite time $(t \rightarrow \infty)$; respectively; $R$ is averaged radius of microsphere; the other symbols are the same as in Equation (1a). In the second instance the expression (4) should be used:

$\frac{M_{t}}{M_{\infty}}=1-\left(\frac{6}{\pi^{2}}\right) \exp \left[-\frac{D_{\beta} \pi^{2} t}{R^{2}}\right]$

The graphic constructions of the diffusion data in the framework of Equation (3) $\left(M_{t} / M_{\infty}-t^{0.5}\right)$ or logarithmic variant of Equation (4) $\left(\ln \left(M_{t} / M_{\infty}\right)-t\right)$ enable us to use these equations as a criterion of transport mechanism and simultaneously calculate the DPD diffusivities in PHB. In Figure 3a the fragments of kinetic profiles for release from PHB microspheres with different diameters are shown. The release data being plotted in coordinates of

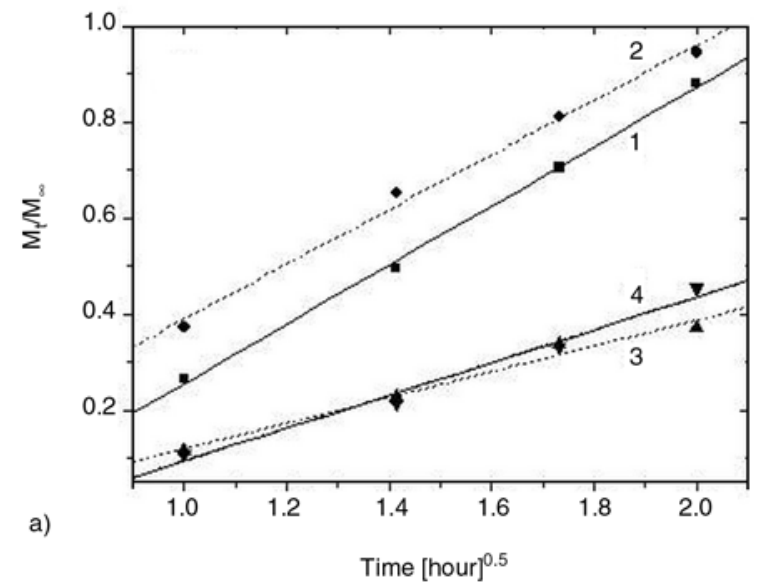

Table 2. DPD diffusion coefficients in the PHB microspheres for relatively small (Equation (3)) and long (Equation (4)) time of diffusion

\begin{tabular}{|c|c|c|}
\hline $\begin{array}{l}\text { Diameter of } \\
\text { specimen } \\
\cdot 10^{3}[\mathrm{~cm}]\end{array}$ & $\begin{array}{c}\text { Diffusion coefficient } \\
\cdot \mathbf{1 0}^{11}\left[\mathrm{~cm}^{2} / \mathrm{s}\right] \\
(\text { Equation }(4))\end{array}$ & $\begin{array}{c}\text { Diffusion coefficient } \\
\cdot \mathbf{1 0}^{11}\left[\mathrm{~cm}^{2} / \mathrm{s}\right] \\
(\text { Equation }(3))\end{array}$ \\
\hline 0,36 & 0,10 & 0,08 \\
\hline 1,9 & 1,5 & 2,0 \\
\hline 6,3 & 2,8 & 2,6 \\
\hline 9,2 & 21,1 & 16 \\
\hline
\end{tabular}

Equation (3) - the relative loss of DPD in microspheres $\left(M_{t} / M_{\infty}\right)$ versus square root of time give the values of diffusion coefficients, $D_{\alpha}=$ $\left.\pi^{2} R^{2} \cdot(\operatorname{tg} \alpha)^{2} / 36\right)$, where $\operatorname{tg} \alpha$ is the slope ratio of linear part of the release curves. The examples of the graphical solution for Equation (4) in semilog coordinates for the microspheres with different diameters are presented in Figure 3b. The integral diffusion coefficients of DPD $\left(D_{\beta}\right)$ could be easily estimated through expression $D_{\beta}=\operatorname{tg} \alpha \cdot R^{2} / \pi^{2}$, where $\operatorname{tg} \beta$ is also the slope ratio of linear part for the release curves presented, however, in the semilog linear coordinates (Figure 3b). Both diffusivities $\left(D_{\alpha}, D_{\beta}\right)$ are shown in Table 2. In case of molecular diffusion one would expect a coincidence between values of diffusion coefficients calculated by different Equations (3) and (4). Table 2 demonstrates actually the closely adjacent values of DPD diffusivities that support the real diffusion mechanism of release going on the initial stage of the kinetics curves. Just as in the paper [21], for the systems PLGA loaded by 5-fluorocyle, in the PHB-DPD samples concerned the diffusion coefficients depend sharply on the size of the microspheres.

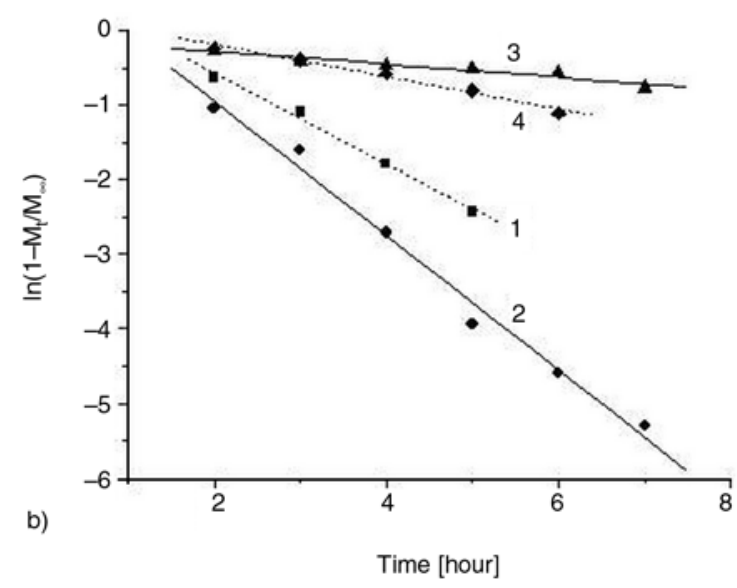

Figure 3. Initial (a) and final (b) regions of diffusion stage for DPD release from the PHB microspheres with diameters $4 \mu \mathrm{m}$ (1), $19 \mu \mathrm{m}$ (2), $63 \mu \mathrm{m}$ (3), and $92 \mu \mathrm{m}$ (4). The data are represented in coordinates of Equation (3) and in semilogarithmic coordinates of Equation (4) 
As previously stated above, the linear kinetics of DPD release occurs together with diffusion mechanism (see Figure 2). For the series of microparticles with diameters 92,63 , and $19 \mu \mathrm{m}$ the kinetic impact comes into particular prominence after terminating diffusion. In contrast to the diffusivity dependence on microsphere size, the constant characteristics $(k)$ of linearity are scarcely affected by diameter of PHB microparticles. The proper rate of hydrolytic degradation should not dependent on geometry of a sample as evidenced by Figure 2 (linear ranges for microspheres with diameters are 92 and $63 \mu \mathrm{m}$ ). The analogous conclusion has been published in the basic paper of Witt and Kissel [26] for PLGA microspheres.

The view of the kinetic profiles as well as the low rate of DPD release are in satisfactory agreement with kinetics of weight loss measured in vitro for the PHB films. The thickness of the PHB films $(40 \mu \mathrm{m})$ was close to the diameters of our microspheres. In this case we assume that the proper hydrolysis kinetics may be similar for both the microspheres and the films.

As is obvious from Figure 4, the change in weight of the films as function of time occurs in accordance with a zero-order equation as well. The exception is an abrupt initial step of weight loss which may be interpreted as a fast extraction of residual impurities. Nature of these impurities is still unknown. It is not impossible that the impurities include the traces of monomers and oligomers

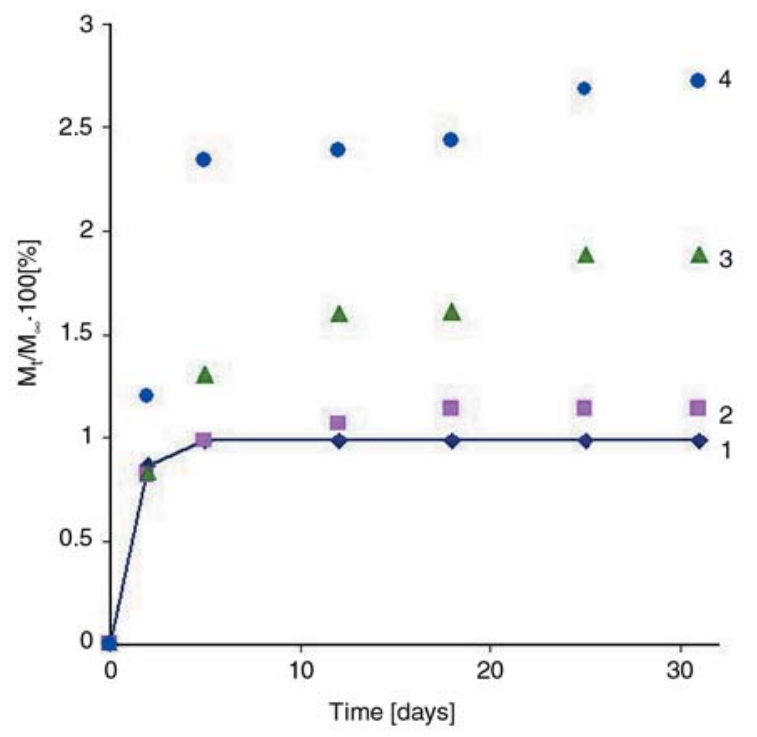

Figure 4. Loss of PHB weight as function of time for the films loaded by DPD. Concentration of DPD: $1(0 \%), 2(3 \%), 3(10 \%)$, and $4(30 \%)$ coming during PHB isolation and film preparation. Whatever the nature of impurities may be, the following slow loss in PHB weight is observed in the same time frame that the linear kinetics of release (compare Figures 2 and 4). Here we note again that the slope of the kinetic curves reflected the weight loss approximately identical. Taking into account the above results, we may suppose that the domination of degradation for both PHB films and microspheres is responsible for the second linear stage of DPD release profiles. Assessment of the changes in the weight of the PHB microspheres immediately and the nature of this phenomenon are an urgent experimental challenge.

\section{Conclusions}

We produced the biodegradable PHB-based microspheres by the oil-in-water emulsion technique. The microspheres with different diameters in interval 4-92 $\mu \mathrm{m}$ were loaded with DPD which presents the class of inhibitors of cell proliferation and simultaneously the antithrombotic drugs. The kinetic profiles of drug release from microspheres into phosphate buffer conformed to the superposition of diffusion and zero-order process of PHB degradation. A sufficiently protensive linear part of kinetic profiles provides a way for application the PHBDPD microsystem as a local drug release medical preparation.

\section{Acknowledgements}

This work was supported by grant no. 06-04-49339 from the Russian Foundation for Basic Research (RFBR) and by grant no. 07-II-10 from Foundation for Assistance to Small Innovative Enterprises (FASIE).

\section{References}

[1] Lonsdale H. K.: The growth of membrane technology. Journal of Membrane Science, 10, 81-181 (1982).

[2] Douglas S. J., Davis S. S., Illum L.: Nanoparticles in drug delivery. CRC Critical Reviews in Therapeutic Drug Carrier Systems, 3, 233-261 (1987).

[3] Langer R. S., Wise D. L.: Medical applications of controlled release, Vol 2, Applications and evaluations. CRC Press, Boca Raton (1984).

[4] Stauch M., Grossmann G., Wanjura D., Adam W-E.: Lack of tolerance after chronic administration of controlled-release isosorbide-5-mononitrate. European Journal of Clinical Pharmacology, 38, Supplement 1, 31-34 (1990). 
[5] Ellis J. T., Kilpatrick D. L., Consigny P. M., Prabhu S., Hossainy S. F. A.: Therapy considerations in drug eluting stents. Critical Reviews in Therapeutic Drug Carrier Systems, 22, 114-139 (2005).

[6] Sarkar K., Sharma S. K., Sachdeva R., Romeo F., Garza L., Mehta J. L.: Coronary artery restenosis: Vascular biology and emerging therapeutic strategies. Expert Review of Cardiovascular Therapy, 4, 543556 (2006).

[7] Masaki T., Kamerath C. D., Kim S-J., Leypoldt J. K., Mohammad S. F., Cheung A. K.: In vitro pharmacological inhibition of human vascular smooth muscle cell proliferation for the prevention of hemodialysis vascular access stenosis. Blood Purification, 22, 307312 (2004).

[8] Singh L. P., Rothfuss K. J., Wiernicki T. R., Lacefield W. B., Kurtz W. L., Brown R. F., Brune K. A., Bailey D., Dube G. P.: Dipyridamole directly inhibits vascular smooth muscle cell proliferation in vitro and in vivo: Implication in the treatment of restenosis after angioplasty. Journal of the American College of Cardiology, 23, 665-671 (1994).

[9] Zhu W., Masaki T., Bae Y. H., Rathi R., Cheung A. K., Kern S. E.: Development of a sustained-release system for perivascular delivery of dipyridamole. Journal of Biomedical Materials Research, Part B: Applied Biomaterials, 77, 135-143 (2006).

[10] Solheim E., Sudmann B., Bang G., Sudmann E.: Biocompatibility and effect on osteogenesis of poly(ortho ester) compared to poly(DL-lactic acid). Journal of Biomedical Materials Research, 49, 257-263 (2000).

[11] Böstman O., Pihlajamäki H.: Clinical biocompatibility of biodegradable orthopaedic implants for internal fixation: a review. Biomaterials, 21, 2615-2621 (2000).

[12] Lickorish D., Chan J., Song J., Davies J. E.: An invivo model to interrogate the transition from acute to chronic inflammation. European Cells and Materials, 8, 12-20 (2004).

[13] Khouw I. M., van Wachem P. B., de Leij L. F., van Luyn M. J.: Inhibition of the tissue reaction to a biodegradable biomaterial by monoclonal antibodies to IFN-gamma. Journal of Biomedical Materials Research, 41, 202-210 (1998).

[14] Su S-H., Nguyen K. T., Satasiya P., Greilich P. E., Tang L., Eberhart R. C.: Curcumin impregnation improves the mechanical properties and reduces the inflammatory response associated with poly(L-lactic acid) fiber. Journal of Biomaterials Science, Polymer Edition, 16, 353-370 (2005).
[15] Bonartsev A. P., Bonartseva G. A., Makhina T. K., Myshkina V. L., Luchinina E. S., Livshits V. A., Boskhomdzhiev A. P., Markin V. S., Iordanskii A. L.: New poly-(3-hydroxybutyrate)-based systems for controlled release of dipyridamole and indomethacin. Applied Biochemistry and Biotechnology, 42, 625630 (2006).

[16] Chen G. Q., Wu Q.: The application of polyhydroxyalkanoates as tissue engineering materials. Biomaterials, 26, 6565-6578 (2005).

[17] Bonartseva G. A., Myshkina V. L., Nikolaeva D. A., Kevbrina M. V., Kallistova A., Gerasin V. A., Iordanskii A. L., Nozhevnikova A. N.: Aerobic and anaerobic microbial degradation of poly- $\beta$-hydroxybutyrate produced by azotobacter chroococcum. Applied Biochemistry and Biotechnology, 109, 285-302 (2003).

[18] Akita S., Einaga Y., Miyaki Y., Fujita H.: Properties of poly( $\beta$-hydroxybutyrate) as a solution. Macromolecules, 9, 774-780 (1976).

[19] Rebrov A. V., Dubinskii V. A., Nekrasov Y. P., Bonartseva G. A., Shtamm M., Antipov E. M.: Structure phenomena at elastic deformation of highly oriented polyhydroxybutyrate. Polymer Science (Russian), 44, 347-351 (2002).

[20] Kassab A. C., Xu K., Denkbas E. B., Dou Y., Zhao S., Piskin E.: Rifampicin carrying PHB microspheres as a potential chemoembolization agent. Journal of Biomaterial Science, Polymer Edition, 8, 947-961 (1997).

[21] Siepmann J., Siepmann F.: Microparticles used as drug delivery systems. Progress in Colloid and Polymer Science, 133, 15-21 (2006).

[22] Kosenko R. Y., Pankova Y. N., Iordanskii A. L., Zaikov G. E.: Controlled release of the antiseptic from poly(3-hydroxybutyrate) films. Combination of diffusion and zero order kinetics. Journal of the Balkan Tribological Association, 13, 242-248 (2007).

[23] Siepmann J., Faisant N., Benoit J-P.: A new mathematical model quantifying drug release from bioerodible microparticles using Monte Carlo simulations. Pharmaceutical Research, 19, 1885-1893 (2002).

[24] Siepmann J., Elkharraz K., Siepmann F., Klose D.: How autocatalysis accelerates drug release from PLGA-based microparticles: A quantitative treatment. Biomacromolecules, 6, 2312-2319 (2005).

[25] Crank J.: The mathematics of diffusion. Clarendon Press, Oxford (1975).

[26] Witt C., Kissel T.: Morphological characterization of microspheres, films and implants prepared from PLGA and ABA triblock copolymers: Is the erosion contolled by degradation, swelling or diffusion? European Journal of Pharmaceutics and Biopharmaceutics, 51, 171-181 (2001). 